\title{
Dynamic Behavior and Its Consideration of EHD Liquid Extraction Phenomenon Causing under DC or Low-Frequency AC Voltage
}

\author{
Ryoichi Hanaoka $^{1}$, Yoji Fujita ${ }^{1}$, Takuma Kajiura ${ }^{2}$ and Hidenobu Anzai ${ }^{3}$ \\ 1. Kanazawa Institute of Technology, 7-1 Ohgigaoka, Nonoichi, Ishikawa 921-8501, Japan \\ 2. Hokkaido Electric Power Co., Inc., 2 Higashi 1-Chome, Odori, Chuo-ku, Sapporo, Hokkaido 060-8677, Japan \\ 3. Fujikura Kasei, Co., Ltd., 5-13-1 Sakurada, Kuki, Saitama 340-0203, Japan
}

\begin{abstract}
This paper describes an experimental and theoretical study on an extraction phenomenon of liquids occurring at an air gap between the liquid surface and the electrode by applying a direct current (DC) or low-frequency alternating current (AC) voltage. Three liquids with a different physical property; 2,3-dihydrodecafluoropenten, palm fatty acid ester oil and crude rapeseed oil are used as working liquids. The electrode configuration is the sphere or plane (high voltage electrode) to grounded plane electrode. The grounded plane electrode is fixed to the bottom of the test vessel with working liquid and the high voltage electrode is installed in an air above the liquid surface against the grounded plane electrode. The liquid surface swells towards the high voltage electrode by the increase of voltage and the liquid is extracted in a short time, thereafter the air gap between the liquid surface and the high voltage electrode is bridged at a thick liquid column. Such the liquid behavior displays unique features with voltage polarity effect for each working liquid. The relationship between the applied voltage, current variation, height of swollen liquid, force pulling liquid and dynamic feature of liquid is examined experimentally. The liquid behavior is considered theoretically based on experimental observations.
\end{abstract}

Key words: Electrohydrodynamics (EHD) extraction phenomenon, semi-insulating and insulating liquids, DC or low-frequency AC voltage, dynamic behavior of liquids, moisture removal effect in oil.

\section{Introduction}

Electrohydrodynamics (EHD) in liquids is a research in the boundary region of electromagnetism and fluid dynamics that deals with the flow phenomenon caused by the interaction between an electric field and a flow field including physicochemical effects. The EHD instability at gas/liquid interface [1, 2], Sumoto effect [3, 4], electrophoretic and dielectrophoretic effects [5], electroosmosis [6, 7] and EHD pumping jet [8-12] are enumerated as typical EHD phenomena and have been studied experimentally and theoretically by many researchers for EHD applications. Furthermore, the

Corresponding author: Ryoichi Hanaoka, professor, research fields: pre-breakdown, breakdown and creeping discharge phenomena in insulating liquids, electric field calculation, EHD phenomena in liquids and development of ER/MR fluids. phenomenon that small droplets are emitted under the action of an electric field is well known as the Taylor cone-jet [13, 14], namely, a conical liquid column (Taylor cone) is formed at the high electric field region of the sharp electrode nozzle and a jet of charged droplets emanates towards a ground electrode (plane electrode etc.) from the tip of the Taylor cone. The flying of droplets is called an electrospray, which has been utilized to an electrospinning apparatus for the production of nanofibers and a mass spectrometer [15, 16]. In this case, a high voltage electrode such as a needle, small sphere, conductive capillary etc. with a sharp tip is required to create a high electric field. On the other hand, there is the phenomenon that the semi-insulating and insulating liquids are extracted towards a higher electric field when a non-uniform electric field is applied to the gas/liquid interface. This 
is referred to as an EHD extraction phenomenon (EHD-EP) [17, 18], which is the target of the present research from a viewpoint of the condensation heat transfer enhancement, flash evaporation, moisture control in liquids etc. [19-21]. The electrode configuration used in this study does not have any sharp edges and the behavior of liquid is opposite to the motion of the above-mentioned Taylor cone-jet. A unified mechanism on an EHD-EP remains to be completely understood, because of a complexity of liquid behavior involving the positive and negative ions, polarized charges, instability at the gas/liquid interface and uniqueness of liquid properties.

In this study, the EHD-EP occurring at different three liquids is observed under a DC or low-frequency AC voltage. The relationship between the applied voltage, current variation, height of swollen liquid, force pulling liquid and dynamic behavior of liquids is measured as experimental data. The fundamental liquid behavior of EHD-EP is considered theoretically based on experimental results.

\section{Experiments}

\subsection{Properties of Working Liquids}

Three liquids with a different physical property; 2,3-dihydrodecafluoropenten (HFC43-10), palm fatty acid ester (PFAE) oil and crude rapeseed (CRS) oil were used as working liquids. The main physical properties of these liquids are shown in Table 1. HFC43-10 is a non-flammable liquid containing fluorine atoms and has an excellent chemical and thermal stability. This liquid is regarded as a semi-insulating liquid, which the electric conductivity has the in-between value (order of $10^{-9} \mathrm{~S} / \mathrm{m}$ ) of an electrolytic solution and a dielectric liquid. The electric conduction is caused by the flow of dissociation ions and indicates the quasi-ohmic characteristics [10-12]. In addition, the ozone depleting potential of HFC-43-10 is zero and the global warming potential is 1,650 , but there is no problem in practical purposes. PFAE oil and CRS oil are a vegetable-based insulating liquid with very low conductivity of the order of $10^{-11}$ $\mathrm{S} / \mathrm{m}$ at low moisture levels of $10 \mathrm{ppm}$ or less, and the dissociation ions in the oil are hard to generate by the electric field. PFAE oil has a saturated fatty acid chemical structure without a double bond between atoms. CRS oil consists of an unsaturated fatty acid chemical structure with a carbon double bond, which contains high percentage of oleic acid. The kinetic viscosity is low in PFAE oil and very high in CRS oil. These oils are better for environmental safety, because of an excellent biodegradability and non-toxicity. However, vegetable-based oils have a high moisture saturation limit $\left(\sim 2,500 \mathrm{ppm}\right.$ at $\left.20{ }^{\circ} \mathrm{C}\right)$ in forms of dissolved, emulsified or free water [22]. The moisture-in-oil control technique is crucial for practical applications of oil, because the moisture content leads to troubles such as acceleration of deterioration, generation of oxide, reduction of equipment life, reduction of dielectric strength etc. The moisture content of PFAE and CRS oils used in this study is of the level of approximately $130-140 \mathrm{ppm}$.

Table 1 Main physical properties of working liquids.

\begin{tabular}{llll}
\hline Main properties & HFC43-10 & PFAE oil & CRS oil \\
\hline Mass density $\left(\mathrm{kg} / \mathrm{m}^{3}\right)$ & $1,585\left(25^{\circ} \mathrm{C}\right)$ & $860\left(20^{\circ} \mathrm{C}\right)$ & $920\left(20^{\circ} \mathrm{C}\right)$ \\
Boiling point $\left({ }^{\circ} \mathrm{C}\right)$ & 55 & None & None \\
Flash point $\left({ }^{\circ} \mathrm{C}\right)$ & None & 176 & 334 \\
Kinetic viscosity $\left(\mathrm{mm}^{2} / \mathrm{s}\right)$ & $0.67\left(25^{\circ} \mathrm{C}\right)$ & $5.10\left(40{ }^{\circ} \mathrm{C}\right)$ & $36.0\left(40{ }^{\circ} \mathrm{C}\right)$ \\
Surface tension $(\mathrm{mN} / \mathrm{m})$ & 14.1 & - & - \\
Relative permittivity & $9.18\left(25^{\circ} \mathrm{C}\right)$ & $2.92\left(80{ }^{\circ} \mathrm{C}\right)$ & $2.86\left(80{ }^{\circ} \mathrm{C}\right)$ \\
Thermal conductivity $\left(25^{\circ} \mathrm{C}\right)(\mathrm{W} / \mathrm{m} \cdot \mathrm{K})$ & 0.075 & 0.13 & 0.18 \\
Electric conductivity $(\mathrm{S} / \mathrm{m})$ & $3.2 \times 10^{-9}\left(25^{\circ} \mathrm{C}\right)$ & $1.4 \times 10^{-11}\left(80^{\circ} \mathrm{C}\right)$ & $2.3 \times 10^{-11}\left(80^{\circ} \mathrm{C}\right)$ \\
\hline
\end{tabular}




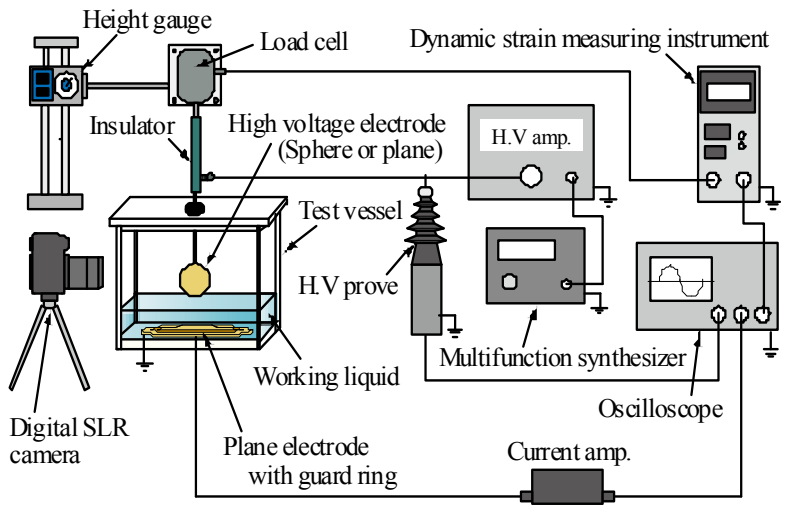

Fig. 1 Schematic of experimental setup employed to observe EHD-EP.

\subsection{Experimental Setup and Procedures}

Fig. 1 shows a schematic of the experimental setup employed to observe the EHD-EP. The brass sphere (diameter: $20 \mathrm{~mm}$ ) or plane (diameter: $24 \mathrm{~mm}$ and thickness: $7 \mathrm{~mm}$ ) was used as a high voltage electrode and was arranged at an interval in the axial direction from the grounded plane electrode. The grounded electrode is a brass flat plate of $48 \mathrm{~mm}$ in diameter and $3.0 \mathrm{~mm}$ in thickness with a guard ring attached around the electrode to accurately measure the current density and is fixed to the bottom of the working liquid inside a sealed cube glass vessel with $75 \mathrm{~mm}$ length, $75 \mathrm{~mm}$ width and $50 \mathrm{~mm}$ height. The spacing between this plane electrode and the liquid surface is maintained in 5 $\mathrm{mm}$. The high voltage electrode is installed in air above the liquid surface, and the air gap $d$ between the liquid surface and the electrode is adjusted to $5 \mathrm{~mm}$. The applied voltage is 0 to $\pm 20 \mathrm{kV}$ DC or a sine wave AC with 0 to $20 \mathrm{kV}$ peak value and frequency of 0.5 to 50 $\mathrm{Hz}$, which is supplied by the multifunction synthesizer (WF 1945A, NF Corp.) and high-voltage power amplifier (30/20A, Trek Japan Co.). When the voltage applying to the electrode is increased, the air/liquid interface swells towards the high voltage electrode along an axis of the electrode system, and the liquid is extracted in a short time. Thereafter, the air gap between the electrode and the liquid is cross-linked by a thick liquid column. These dynamic behaviors for each working liquid were recorded as an animation by using the digital single-lens reflex (SLR) camera (D-750, Nikon Co.) and personal computer. The height of the swollen liquid can be examined from these photographic data. The force pulling liquid was measured by a combination of the micro-load lord cell (LTS-500GA, 5N) installed to the high voltage electrode through a solid insulator and the dynamic strain measuring instrument (Strain Amplifire; DPM-911B, Kyowa Electronic Instruments Co. Ltd.). The current variation between the high voltage electrode and the grounded electrode was observed by the variable gain low-noise current amplifier (DLPCA-200, Femto Messtechnik $\mathrm{GmbH}$ ) and digital oscilloscope connected to the grounded electrode. The experiment was carried out at room temperature and atmospheric pressure.

\section{Experimental Results and Discussion}

\subsection{Behavior of Liquids and Dynamic Patterns}

Fig. 2 shows typical pictures of the EHD-EP in three working liquids occurring at the peak value $V_{0}$ in positive and negative half-cycles of the AC voltage with frequency $f=0.5 \mathrm{~Hz}$. The distinctive behavior was observed for each working liquid. The liquid at air/liquid interface swells gradually towards the high voltage electrode along an axis of the electrode system with the increase of $V_{0}$ as shown in Figs. 2A, 2B and $2 \mathrm{C}$-a. The swelling area of the liquid is larger at the plane electrode with a wider electric field area than the sphere electrode. At a certain value of $V_{0}$, the liquid is suddenly extracted from the swollen surface in the form of droplets or liquid thread as shown in Figs. 2A, $2 \mathrm{~B}$ and $2 \mathrm{C}-\mathrm{b}$. Such the liquid extraction occurs from the tip of the swollen liquid in the case of the sphere electrode and from anywhere in the swollen flat surface in the case of the plane electrode. Thereafter, with a slight increase in $V_{0}$, the high voltage electrode and the liquid are bridged by the thick liquid column, and the thickness of liquid column increases with increasing $V_{0}$. There was a clear difference in the behavior of liquid between HFC43-10 and PFAE (or CRS) oil after the 
liquid column formation. HFC43-10 indicates active behavior with a voltage polarity effect. In a positive half-cycle of the voltage, many fine showers of liquid come violently down from the electrode surface to the liquid surface nearly along a line of electric force as shown in Fig. 2A-c, d, e. Such the shower is very poor at a negative half-cycle of the voltage. The liquid behavior in a positive half-cycle is very active compared to that in a negative half-cycle. The inside of the liquid column is in a turbulent flow state. HFC43-10 is recognized as a semi-insulating liquid with a quasi-ohmic electrical conduction property dominated by dissociated ions due to the electric field. Positive and negative dissociation ions are produced in a pair, but in HFC43-10 with many atoms of C, H, F, O, because the mass of ion species is different for positive and negative ions, their mobility will be different. It has been guessed that the mobility of dissociation ions in

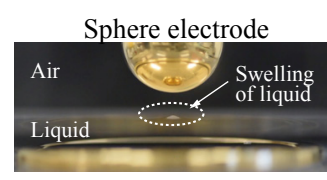

(a) $V_{0}=+8.4 \mathrm{kV}$

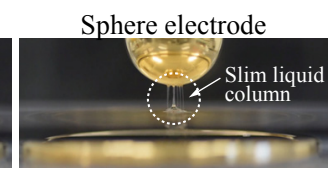

(b) $V_{0}=+8.7 \mathrm{kV}$

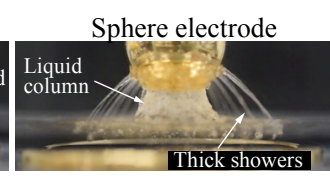

(c) $V_{0}=+14.5 \mathrm{kV}$

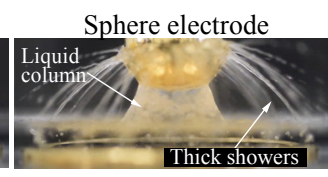

(d) $V_{0}=+20.0 \mathrm{kV}$

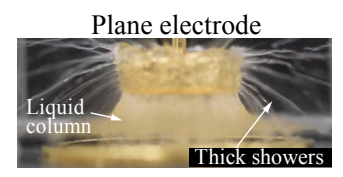

(e) $V_{0}=+20.0 \mathrm{kV}$

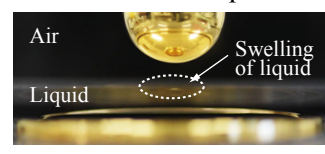

(a) $V_{0}=-8.4 \mathrm{kV}$

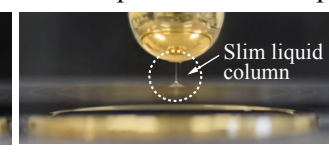

(b) $V_{0}=-8.7 \mathrm{kV}$

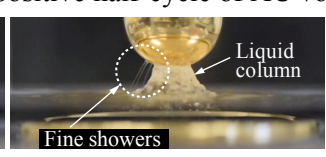

(c) $V_{0}=-14.5 \mathrm{kV}$

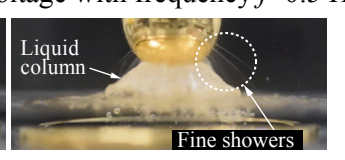

(d) $V_{0}=-20.0 \mathrm{kV}$

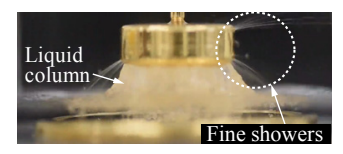

(e) $V_{0}=-20.0 \mathrm{kV}$

Liquid behaviors at peak value $V_{0}$ in negative half-cycle of $\mathrm{AC}$ voltage with frequency $f=0.5 \mathrm{~Hz}$

(A) HFC43-10

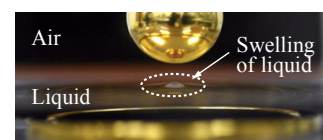

(a) $V_{0}=+10.2 \mathrm{kV}$

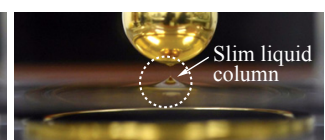

(b) $V_{0}=+10.6 \mathrm{kV}$

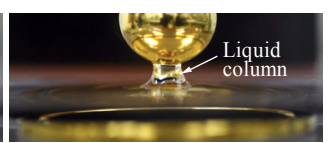

(c) $V_{0}=+14.5 \mathrm{kV}$

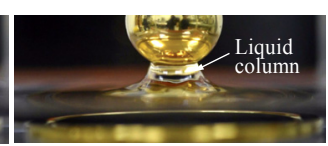

(d) $V_{0}=+20.0 \mathrm{kV}$

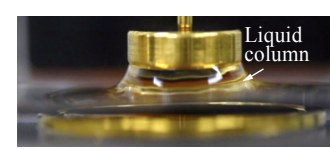

(e) $V_{0}=+20.0 \mathrm{kV}$

Liquid behaviors at peak value $V_{0}$ in positive half-cycle of AC voltage with frequency $f=0.5 \mathrm{~Hz}$

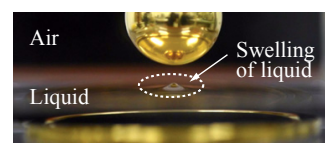

(a) $V_{0}=-10.2 \mathrm{kV}$

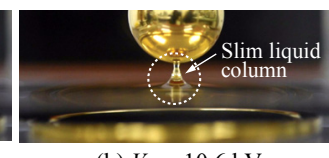

(b) $V_{0}=-10.6 \mathrm{kV}$

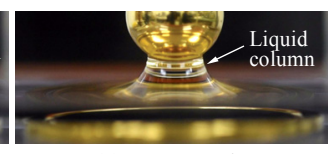

(c) $V_{0}=-14.5 \mathrm{kV}$

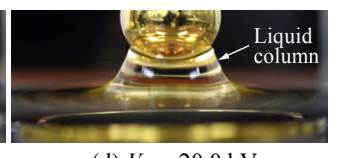

(d) $V_{0}=-20.0 \mathrm{kV}$

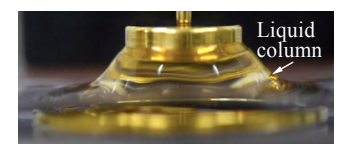

(e) $V_{0}=-20.0 \mathrm{kV}$

Liquid behaviors at peak value $V_{0}$ in negative half-cycle of AC voltage with frequency $f=0.5 \mathrm{~Hz}$

(B) PFAE oil

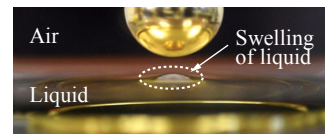

(a) $V_{0}=+14.5 \mathrm{kV}$

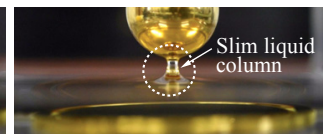

(b) $V_{0}=+14.9 \mathrm{kV}$

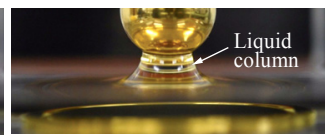

(c) $V_{0}=+16.0 \mathrm{kV}$

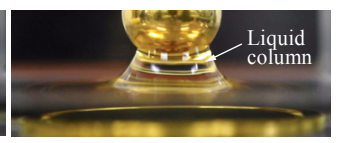

(d) $V_{0}=+20.0 \mathrm{kV}$

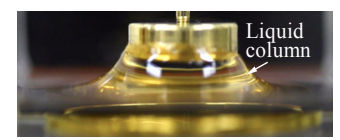

(e) $V_{0}=+20.0 \mathrm{kV}$

Liquid behaviors at peak value $V_{0}$ in positive half-cycle of AC voltage with frequency $f=0.5 \mathrm{~Hz}$

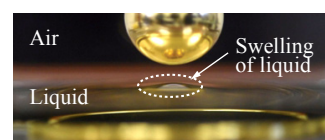

(a) $V_{0}=-14.5 \mathrm{kV}$

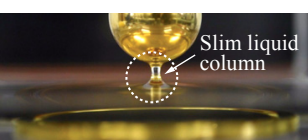

(b) $V_{0}=-14.9 \mathrm{kV}$

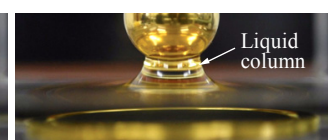

(c) $V_{0}=-16.0 \mathrm{kV}$

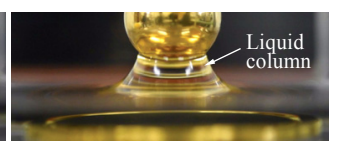

(d) $V_{0}=-20.0 \mathrm{kV}$

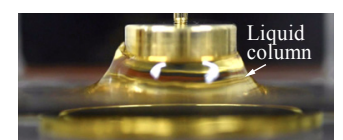

(e) $V_{0}=-20.0 \mathrm{kV}$

Liquid behaviors at peak value $V_{0}$ in negative half-cycle of AC voltage with frequency $f=0.5 \mathrm{~Hz}$

(C) CRS oil

Fig. 2 Typical behaviors of EHD-EP in three working liquids (air gap $d=5 \mathbf{m m}$ ). 
HFC43-10 is $\sim 1.0 \times 10^{-8} \mathrm{~m}^{2} / \mathrm{V} \cdot \mathrm{s}$ for negative ions and $\sim 0.7 \times 10^{-8} \mathrm{~m}^{2} / \mathrm{V} \cdot \mathrm{s}$ for positive ions [12]. This suggests that the effect of negative ion species with large mobility will appear intensely to the liquid behavior under the positive applied voltage.

The behavior in PFAE oil and CRS oil is different from the phenomenon in HFC43-10. The shower phenomenon confirmed in HFC43-10 does not occur with these liquids as shown in Figs. 2B and 2C-c, d, e. The liquid column in which the air gap is cross-linked is formed at a voltage higher than that of HFC43-10. The thickness of liquid column in PFAE oil is thicker in a negative half-cycle than a positive half-cycle of the voltage as shown in Fig. 2B-c, d, e. This voltage polarity effect on the liquid activity is opposite to that in HFE43-10. PFAE oil is the vegetable-based insulating liquid which is derived by the synthesized process involved with transesterification of fatty acid methyl ester and alkyl alcohol through molecular design technique and has the chemical structure of fatty acid alkyl esters (RCOOR). Thereby, the functional group may be positively charged by the action of the electric field. The effect of the positive charges will appear intensely to the liquid behavior under the negative applied voltage. In contrast, the liquid column pattern of CRS oil is much the same in positive and negative half-cycles of the voltage as shown in Fig. $2 \mathrm{C}-\mathrm{c}, \mathrm{d}$, e. CRS oil is the vegetable insulating liquid that the dissociation ions are hard to generate by the electric field. The charge generation in this case will be based on the polarization effect of liquid molecules or impurities. Because positive and negative polarization charges are equivalent, there is no voltage polarity effect on the liquid activity. These behaviors of the liquid are repeated periodically for each positive and negative half-cycle of an $\mathrm{AC}$ voltage according to the voltage value and are sustained at a constant shape for a DC voltage. However, the liquid activities depend on the frequency of AC voltage. Typical examples of the frequency dependence of liquid behavior are shown in Fig. 3. Since the movement of liquid can not follow the rising frequency, the activity of liquid decays with increasing frequency (especially, in CRS oil with high kinetic viscosity).

\subsection{Current Variation, Height of Swollen Liquid and} Force Pulling Liquid

When a sine wave $\mathrm{AC}$ voltage is applied to the high voltage electrode, the electrode is subjected to the force bringing out the liquid in response to the instantaneous value and frequency of the voltage. Fig. 4 shows typical relationships between the applied voltage $V_{(t)}$,

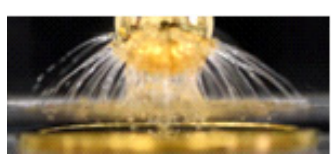

(a) DC

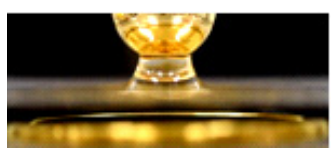

(a) DC

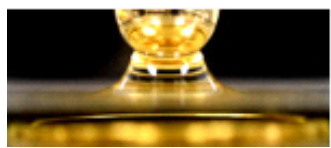

(a) DC

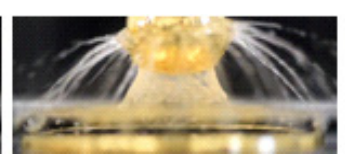

(b) AC: $f=0.5 \mathrm{~Hz}$

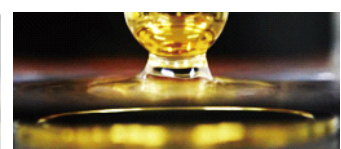

(b) AC: $f=0.5 \mathrm{~Hz}$

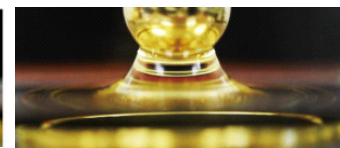

(b) $\mathrm{AC}: f=0.5 \mathrm{~Hz}$

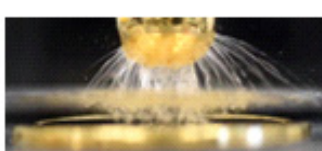

(c) $\mathrm{AC}: f=5.0 \mathrm{~Hz}$

(A) HFC43-10

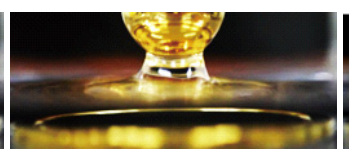

(c) $\mathrm{AC}: f=5.0 \mathrm{~Hz}$

(B) PFAE oil

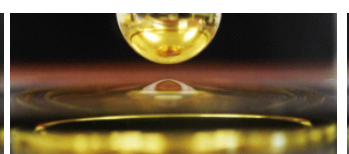

(c) $\mathrm{AC}: f=5.0 \mathrm{~Hz}$

(C) CRS oil

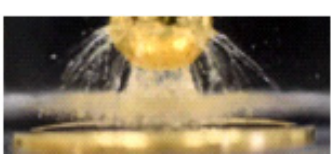

(d) $\mathrm{AC}: f=10.0 \mathrm{~Hz}$

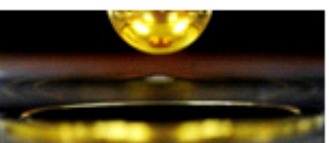

(d) AC: $f=10.0 \mathrm{~Hz}$

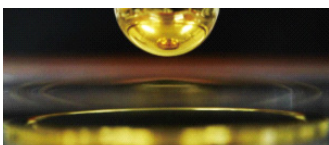

(d) AC: $f=10.0 \mathrm{~Hz}$

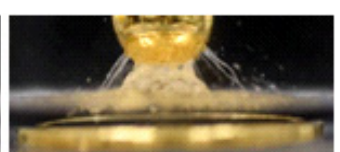

(e) $\mathrm{AC}: f=50.0 \mathrm{~Hz}$

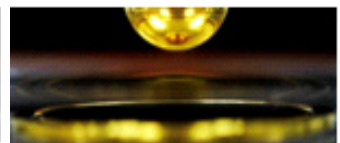

(e) $\mathrm{AC}: f=50.0 \mathrm{~Hz}$

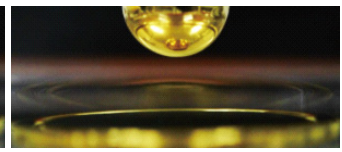

(e) $\mathrm{AC}: f=50.0 \mathrm{~Hz}$

Fig. 3 Frequency dependence of liquid behavior (sphere electrode, $d=5 \mathrm{~mm}$, positive voltage $V_{0}=+20 \mathrm{kV}$ ). 


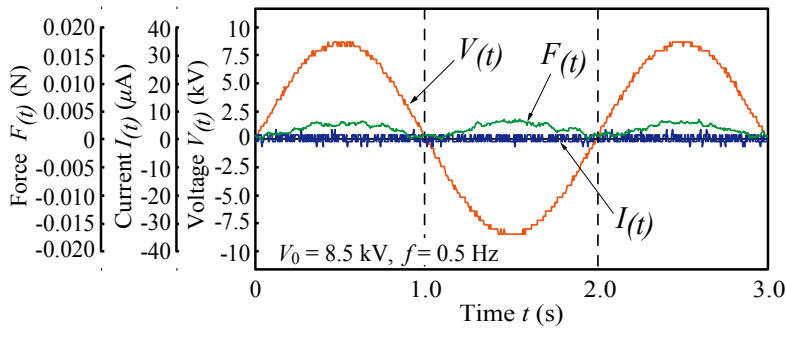

(a) HFC43-10 (before liquid column formation)

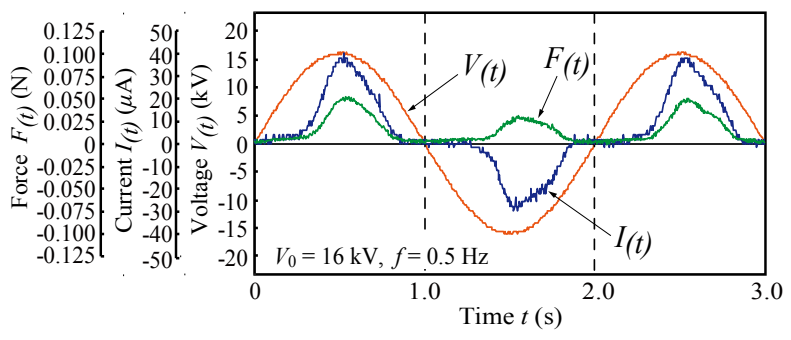

(b) HFC43-10 (after liquid column formation)

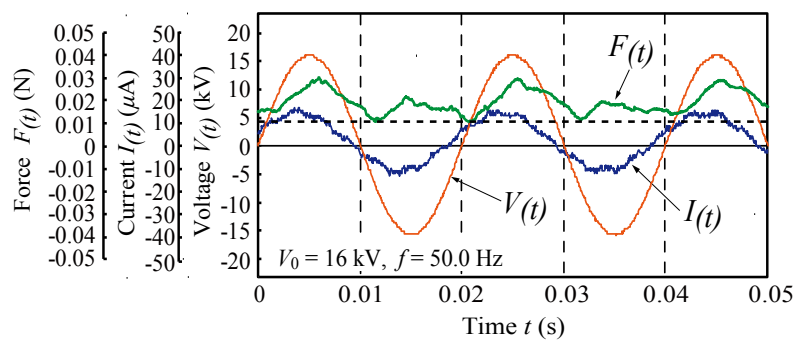

(c) HFC43-10 (after liquid column formation)

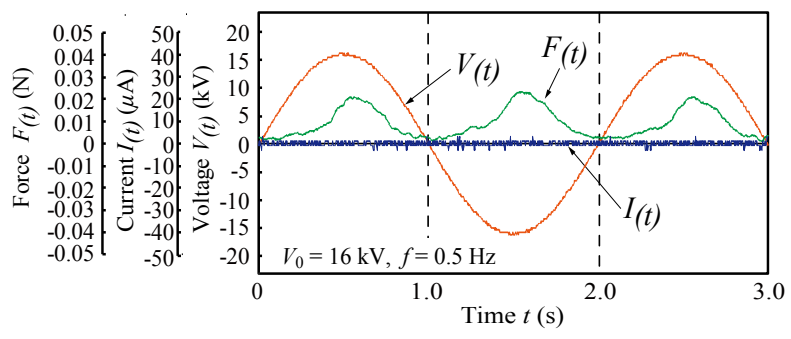

(d) PFAE oil (after liquid column formation)

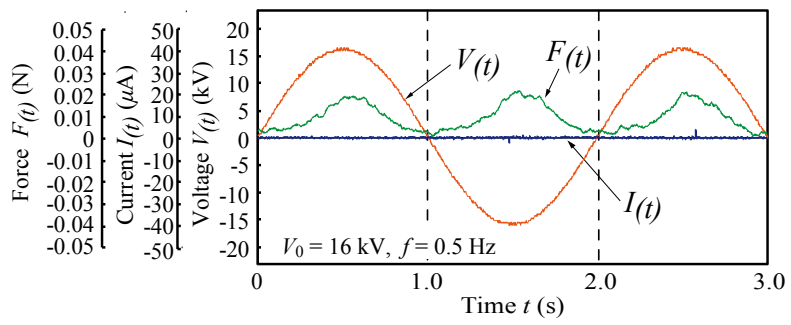

(e) CRS oil (after liquid column formation)

Fig. 4 Relationships between $V_{(t)}, I_{(t)}$ and $F_{(t)}$.

current variation $I_{(t)}$ and force pulling liquid $F_{(t)}$ before and after the formation of liquid column. The current can not be detected before the liquid column formation whatever the liquid (see Fig. 4a). The current in
HFE43-10 flows by the formation of liquid column (see Fig. 4b), but the current in both PFAE oil and CRS oil can not be detected even if the air gap is bridged by the liquid column (see Figs. $4 \mathrm{~d}$ and $4 \mathrm{e}$ ), because of a high insulation property of their liquids. Furthermore, when the frequency $f$ increases to exceed $\sim 20 \mathrm{~Hz}$ for HFC43-10, $\sim 5 \mathrm{~Hz}$ for PFAE oil and $\sim 2 \mathrm{~Hz}$ for CRS oil, the force pulling liquid $F_{(t)}$ can not return to zero at the zero point of a voltage sine wave as shown by the dotted line in Fig. 4c, because the liquid is incapable of following a change of the frequency.

Fig. 5 shows the relationship between the maximum height of swollen liquid $\Delta h_{0}$ and the peak value of voltage $V_{0}(f=0.5 \mathrm{~Hz})$ before the liquid column formation, which is obtained from animation data of the liquid behavior. Fig. 6 shows the relationship between the force pulling liquid $F_{0}$ and the $V_{0}$ $(f=0.5 \mathrm{~Hz})$ before and after the formation of liquid column, where $F_{0}$ denotes the peak value of force variations. In HFC43-10 and PFAE oil, the opposite voltage polarity effect can be confirmed in the curves of $\Delta h_{0}$ and $F_{0} . \Delta h_{0}$ at the plane electrode is slightly higher than that at the sphere electrode under identical $V_{0}$ (as seen at HFC43-10). The force $F_{0}$ increases steeply after the liquid column formation. $F_{0}$ at the plane electrode is much larger than that at the sphere electrode under identical $V_{0}$ (as seen at HFC43-10).

\subsection{Analytical Consideration of Liquid Behavior}

The liquid behavior in this study can be analytically considered based on the experimental observation.

The liquid swelling model before the liquid column formation is shown in Fig. 7 using a sphere electrode. When a sine wave AC voltage $V_{(t)}$ which is a function of time $t$ is applied to the sphere electrode, the charges $Q_{(t)}$ of opposite polarity to the electrode are accumulated at the air/liquid interface. Then the electrode attracts the liquid with force $F_{(t)}$ and lifts the liquid to height $\Delta h_{(t)}$ by the balance of the $F_{(t)}$ and the downward force (the sum of the forces due to the gravitation and surface 


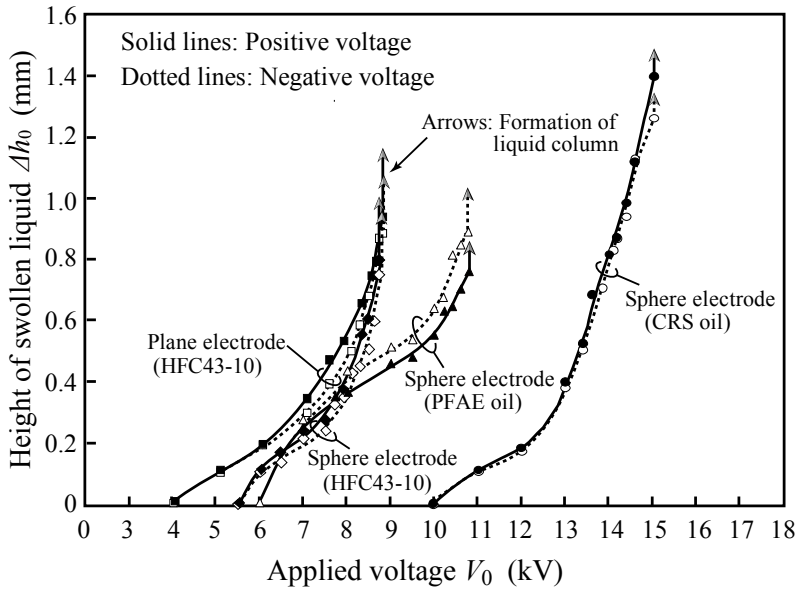

Fig. 5 Relationship between $\Delta h_{0}$ and $V_{0}(f=0.5 \mathrm{~Hz})$.

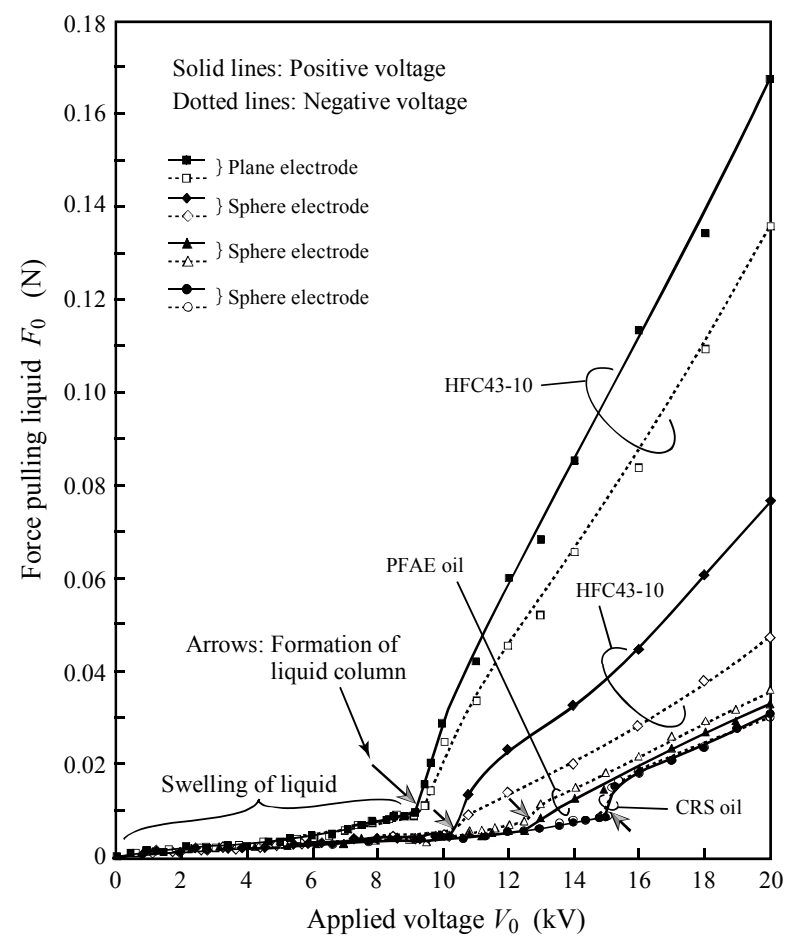

Fig. 6 Relationship between $F_{0}$ and $V_{0}(f=0.5 \mathrm{~Hz})$.

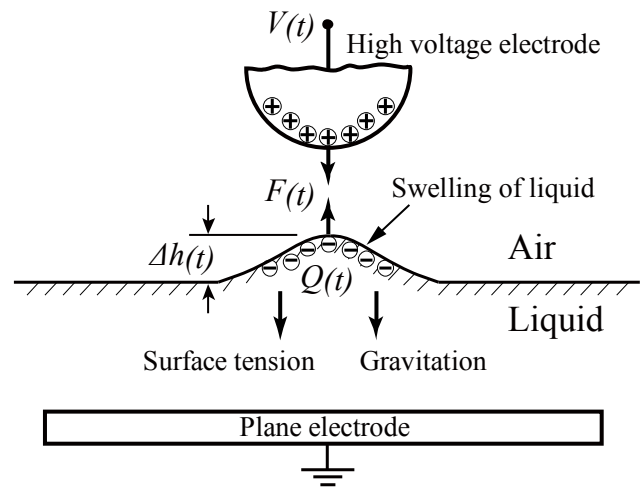

Fig. 7 Swelling model of liquid for sphere electrode. tension). The time variation model of the voltage $V_{(t)}$, force pulling liquid $F_{(t)}$ and height of swollen liquid $\Delta h_{(t)}$ is described as Fig. 8.

The applied voltage is $V_{(t)}=V_{0} \sin \omega t$ for a sine wave AC voltage, where $V_{0}$ is the peak value, $\omega$ the angular frequency and $t$ the time. Thus electric field $E_{(t)}$ in the gap between the electrodes also changes in a sine curve with time as follows.

$$
E_{(t)}=E_{0} \sin \omega t
$$

where $E_{0}$ is the peak value of electric field. The force pulling liquid $F_{(t)}$ represents the attractive force acting between the charge accumulated to air/liquid interface and the charge induced on electrode surface. The time variation of $F_{(t)}$ is expressed as follows from the model of Fig. 8.

$$
F_{(t)}=\left(F_{0} / 2\right)(1-\cos 2 \omega t)+\alpha
$$

where the peak value of the force occurring at each half-cycle of the $V_{(t)}$ is $F_{0}+\alpha$; $\left(F_{01}+\alpha\right.$ or $\left.F_{02}+\alpha\right)$ and $\alpha$ denotes the residual force component that occurs as the frequency increases. That is, as described in Section $3.2, \alpha$ is approximately zero for low frequencies and has a finite value with increasing frequency. The liquid swells to a certain height $\Delta h_{(t)}$ from the air/liquid interface by the effect of $F_{(t)}$. The time variation of $\Delta h_{(t)}$ is synchronized with the variation of $F_{(t)}$, and it is expressed as follows from the model of Fig. 8.

$$
\Delta h_{(t)}=\left(\Delta h_{0} / 2\right)(1-\cos 2 \omega t)+\beta
$$

where the peak value of the height occurring at each half-cycle of the $V_{(t)}$ is $\Delta h_{0}+\beta ;\left(\Delta h_{01}+\beta\right.$ or $\left.\Delta h_{02}+\beta\right)$ and $\beta$ denotes the residual swelling height component that occurs as the frequency increases. $\beta$ is approximately zero for low frequencies and has a finite value with increasing frequency. The value of $F_{(t)}$ can be obtained from the variation waveform of the force (see Fig. 4). The value of $\Delta h_{(t)}$ can also be estimated by analyzing photographic data obtained from the animation of the liquid behavior. The force $F_{(t)}$ will be generated according to Coulomb's law which acts on opposite polarity charges $\pm Q_{(t)}$ of the electrode and the liquid surface as expressed by the following equation.

$$
F_{(t)}=\left|-1 / 4 \pi \varepsilon_{0}\right|\left\{Q_{(t)}{ }^{2} /\left(d-\Delta h_{(t)}\right)^{2}\right\}
$$




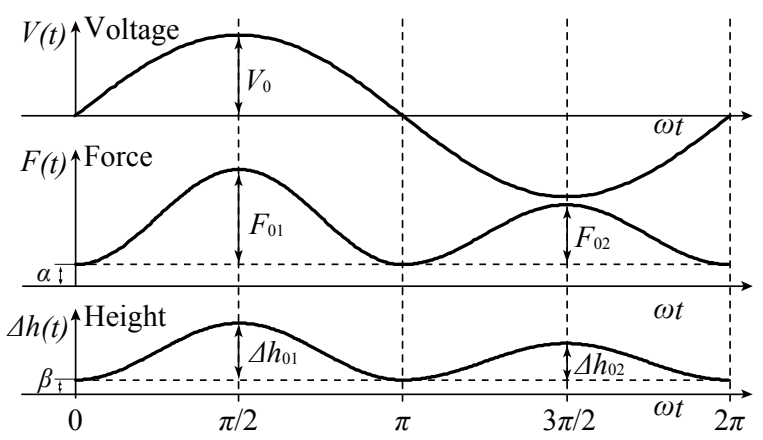

Fig. 8 Time variation model of $V_{(t)}, F_{(t)}$ and $\Delta h_{(t)}$ for AC voltage.

where $d$ denotes the distance between the electrode surface and the liquid surface and $\varepsilon_{0}$ the permittivity of air. Therefore, the charge $Q_{(t)}$ at the air/liquid interface is expressed as follows from Eqs. (2) and (4).

$$
\begin{aligned}
Q_{(t)}= & 2\left(\pi \varepsilon_{0}\right)^{0.5}\left[d-\left\{\left(\Delta h_{0} / 2\right)(1-\cos 2 \omega t)+\beta\right\}\right] \\
& \times\left\{\left(F_{0} / 2\right)(1-\cos 2 \omega t)+\alpha\right\}^{0.5}
\end{aligned}
$$

In the situation that the liquid is pulled up to the $\Delta h_{0}+\beta$, the Coulomb force acting on the charge $Q_{(t)}$ at the air/liquid interface in the electric field balances with the downward force which is the sum of the forces due to the gravitation and surface tension. This is expressed as follows.

$$
Q_{(t)} E_{(t)}=\rho g v_{s(t)}+\gamma \nabla^{2} \Delta h_{(t)} S_{(t)} \equiv F_{(t)}
$$

In Eq. (6), the left-hand side denotes the Coulomb force. The first term on the right-hand side denotes the gravitational force where $\rho$ is the density of liquid, $g$ the gravitational acceleration and $v_{s(t)}$ the volume of swollen liquid, and the second term denotes the force due to the surface tension where $\gamma$ is the surface tension of liquid, $\Delta h_{(t)}$ the height of swollen liquid and $S_{(t)}$ the surface area of swollen liquid. This Coulomb force will be identical to the $F_{(t)}$. Thus, the electric field $E_{(t)}$ necessary to pull up the liquid is expressed as follows from Eqs. (1), (2) and (6).

$$
E_{(t)}=\left(1 / Q_{(t)}\right)\left\{\left(F_{0} / 2\right)(1-\cos 2 \omega t)+\alpha\right\} \equiv E_{0} \sin \omega t
$$

By the way, Maxwell's stress $T_{(t)}$ comparable to the force $F_{(t)}$ is acting on the surface area $S_{(t)}$ as expressed by the following equation.

$$
T_{(t)}=(1 / 2) \varepsilon_{0} E_{(t)}^{2} S_{(t)} \equiv F_{(t)}
$$

Thus, $S_{(t)}$ is

$$
S_{(t)}=2 F_{(t)} / \varepsilon_{0} E_{(t)}^{2}
$$

If the shape of the swollen liquid for the sphere electrode is roughly assumed to be a conical shape with the height of $\Delta h_{(t)}$ and the bottom of diameter $D_{(t)}, S_{(t)}$ is equal to the lateral area of the cone, thereby $D_{(t)}$ is expressed as follows.

$$
D_{(t)}=2^{0.5}\left[-\Delta h_{(t)}{ }^{2}+\left\{\Delta h_{(t)}{ }^{4}+\left(4 F_{(t)} / \pi \varepsilon_{0} E_{(t)}{ }^{2}\right)^{2}\right\}^{0.5}\right]^{0.5}
$$

Since the circle area of diameter $D_{(t)}$ is the region where the liquid swells, the volume $v_{s(t)}$ of the swollen liquid is

$$
v_{s(t)}=(1 / 3) \pi\left(D_{(t)} / 2\right)^{2} \Delta h_{(t)}
$$

On the other hand, when the voltage reaches a critical value, the air gap between the electrode and the liquid surface is cross-liked by a thick liquid column with a nearly cylindrical shape. In this case, a force pulling liquid will balance with the gravitational force. The volume $v_{c(t)}$ of liquid column at that time is expressed as follows.

$$
v_{c(t)}=F_{(t)} / \rho g
$$

These analyses can also be performed similarly as $V_{(t)}=V_{0}, E_{(t)}=E_{0}, F_{(t)}=F_{0}$ and $\Delta h_{(t)}=\Delta h_{0}$ when a DC voltage that is not a function of time is applied to the electrodes. And also, when an AC voltage with low frequency (for instance, $f=0.5 \mathrm{~Hz}$ ) is applied, the components of $\alpha$ and $\beta$ are almost zero.

According to the above rough analysis, the electric field and charge required for an EHD-EP before the liquid column formation can be estimated as a function of voltage. For instance, in the case that a sine wave AC voltage $V_{(t)}$ with the frequency of $0.5 \mathrm{~Hz}$ is applied to the sphere electrode, the electric field $E_{0}$ and charge $Q_{0}$ obtained in the $\omega t=\pi / 2$ at positive half-cycle and $\omega t=3 \pi / 2$ at negative half-cycle of the $V_{(t)}$ are shown in Fig. 9. For all working liquids, the values of $E_{0}$ and $Q_{0}$ are in the range of $\sim 1.0-2.3 \mathrm{MV} / \mathrm{m}$ and $\sim 2.5-3.5 \mathrm{nC}$, respectively. The diameter $D_{0}$ of the swollen liquid under identical $\omega t$ is nearly comparable to the diameter of sphere electrode; the region where electric field acts.

\subsection{Moisture-in-Oil Removal Effect of EHD-EP}

The study on evaporative heat transfer utilizing the EHD effect has been reported by several researchers 


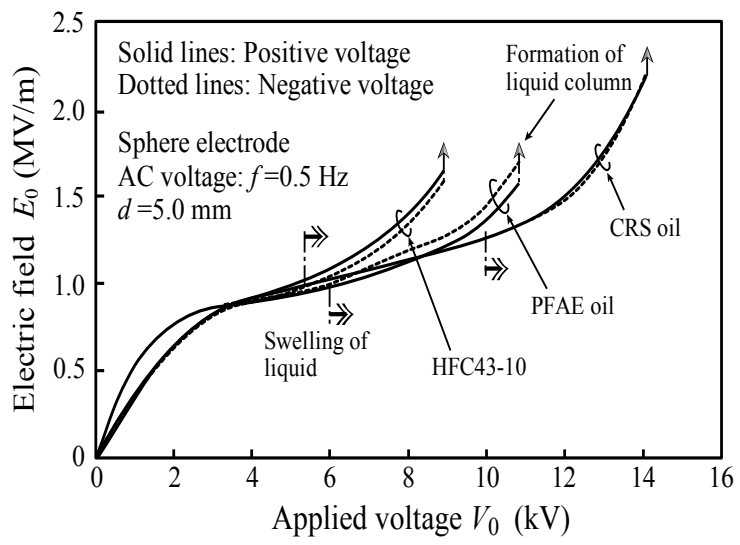

(a) Relationship between $E_{0}$ and $V_{0}$

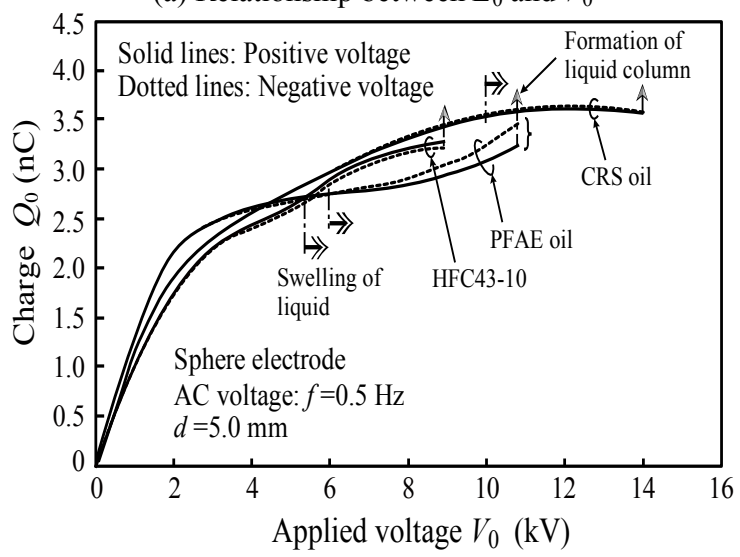

(b) Relationship between $Q_{0}$ and $V_{0}$

Fig. 9 Typical example of $E_{0}$ and $Q_{0}$ required for EHD-EP before liquid column formation.

[19, 20, 23-25]. In these reports, the source of liquid swelling caused by the electric field has been considered to be due to the occurrence of EHD instability at the air/liquid interface and it has been confirmed that the evaporation of droplets can be enhanced by the EHD-EP. The swollen liquid shifts from the extraction of droplets (or liquid thread) to the formation of liquid column by increasing applied voltage. Now, it is examined experimentally whether the evaporation of moisture in the oil is promoted by sustaining the state after the liquid column formation for a long time. Fig. 10 shows a schematic of the experimental setup used to confirm the moisture evaporation in oil. In this experiment, CRS oil was used as the test oil. At first, the test oil is exposed to the boiling saturated vapor of distilled water in a desiccator and the moisture content is forcibly adjusted to 500 ppm by using a Karl Fisher titration. In this case, the moisture in CRS oil will be able to exist as emulsified or free water rather than dissolved water. Fifty (50) $\mathrm{mL}$ of moisture-containing sample oil was introduced into an acrylic cylindrical test vessel. The electrode arrangement is basically the same as Fig. 1, but the plane electrode with $30 \mathrm{~mm}$ in diameter is used as a high voltage electrode and it is installed in air at a distance of $d=5 \mathrm{~mm}$ from the liquid surface. The spacing between the grounded plane electrode (54.5 $\mathrm{mm}$ in diameter with a guard ring) in the liquid and the liquid surface is maintained in $5 \mathrm{~mm}$. Air in the test vessel is circulated through a vinyl pipe connecting the molecular sieve box and diaphragm pump in series as shown in Fig. 10. Thus, moisture in the air is absorbed by the molecular sieve. The oil temperature $T$ can be controlled by a flexible sheathed heater wrapped around the test vessel and is measured by a thermocouple thermometer. DC negative voltage of 20 $\mathrm{kV}$ is applied to the high voltage electrode. As soon as a voltage is applied to the electrode, a liquid column covering the electrode surface is formed as shown in Fig. 2C-e. The pattern of liquid column is sustained during the application of voltage. The moisture content in the sampling oil is measured by a Karl Fisher titration at any given time. The typical variations with time of the moisture content are shown in Fig. 11. In this experimental method, the moisture decreases with time even if the voltage is zero (no EHD-EP). This indicates that the evaporation of moisture is due to the circulation of dry air. However, Fig. 11 shows that the evaporation rate of moisture is greatly promoted by the occurrence of EHD-EP, and this effect becomes more remarkable as the liquid temperature is raised. Water droplets with a large permittivity in oil have a large polarization effect due to an electric field and are drawn towards the air/oil interface by the Coulomb force. It is seemed that the polarized water droplets at the interface are subjected to the effect of being drawn out into the air by Maxwell's stress. The oil temperature will promote the activation of water molecules. 


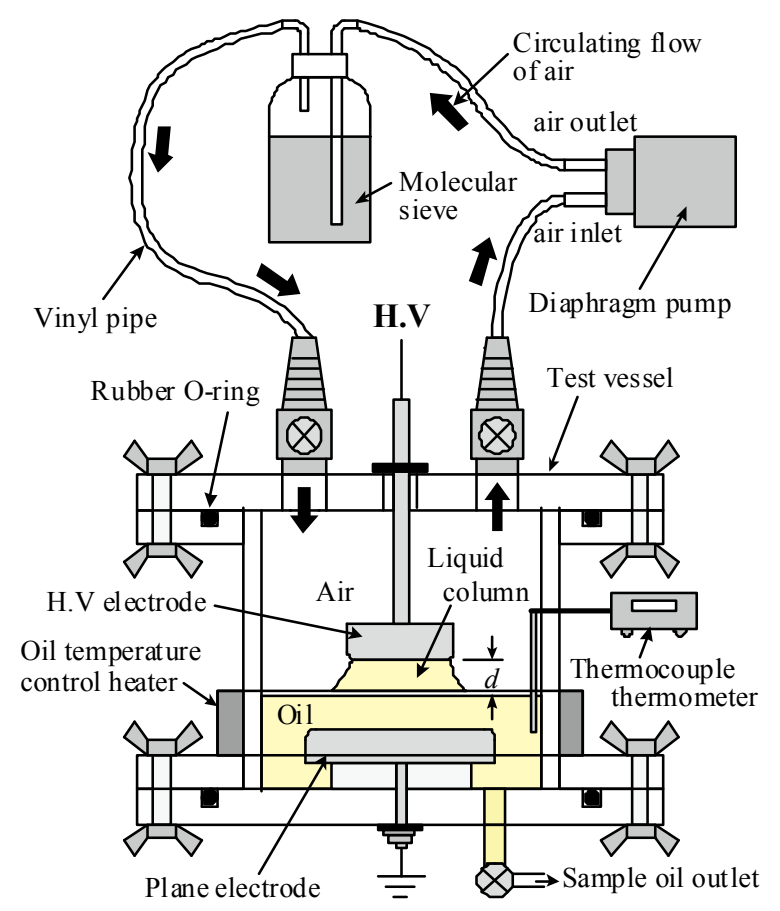

Fig. 10 Schematic of experimental setup used to confirm moisture evaporation in oil.

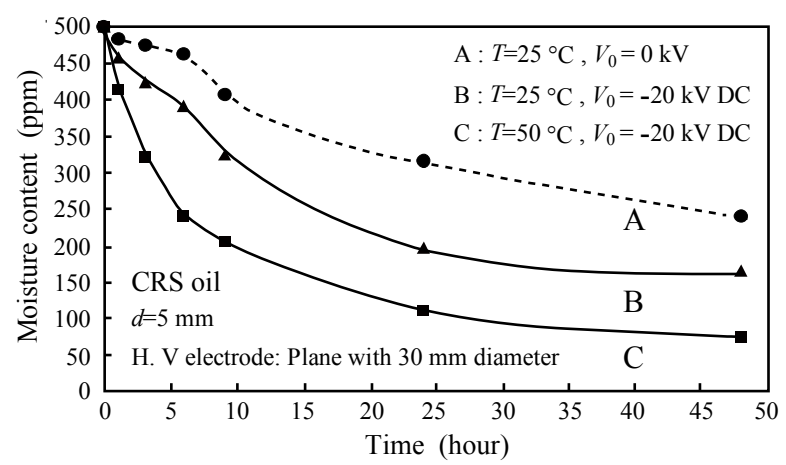

Fig. 11 Typical variation with time of moisture content.

\section{Conclusions}

The EHD-EP generating at an air gap between the high voltage electrode (sphere or plane electrode) and the liquid surface was investigated under a DC or low-frequency $\mathrm{AC}$ voltage using three liquids with a different physical property; HFC-43-10, PFAE oil and CRS oil. A distinctive behavior of liquid in quasiuniform electric fields was observed depending on the type of liquid and the voltage polarity. The liquid is suddenly extracted in the form of droplets or liquid thread from the swollen surface after swelling to a height of $\sim 0.8-1.4 \mathrm{~mm}$ (for $5.0 \mathrm{~mm}$ air gap) with the rise of voltage. Thereafter, the air gap is connected by a thick liquid column with a slight increase in voltage. The thickness of the liquid column depends on the voltage value. The force pulling liquid increases steeply after the formation of liquid column and the force at the plane electrode is much larger than that at the sphere electrode under identical voltage.

In HFC43-10, the shower phenomenon which comes down from the high voltage electrode surface to the liquid surface is observed immediately after the liquid column formation. The occurrence of showers is much more active in the positive voltage than negative voltage. Such the shower phenomenon does not occur with PFAE oil and CRS oil and the liquid column is formed at a voltage higher than that of HFC43-10. The thickness of liquid column in PFAE oil is thicker in the negative voltage than positive voltage, but in CRS oil, there is no voltage polarity effect on the liquid column pattern.

The liquid behavior of EHD-EP was roughly considered from the principle of electromagnetism based on the experimental observation results. The analytical results reveal that electric fields and charges in the range of $\sim 1.0-2.3 \mathrm{MV} / \mathrm{m}$ and $\sim 2.5-3.5 \mathrm{nC}$ are required for the occurrence of EHD-EP, respectively. On the other hand, it was experimentally proved that the moisture evaporation in oil was enhanced by EHD-EP. The results obtained from this study will provide a notable indicator for engineers involved in EHD applications.

\section{References}

[1] Melcher, R. 1981. Continuum Electromechanics. The MIT Press.

[2] Atten, P. 1996. "Electrohydrodynamic Instability and Motion Induced by Injected Space Charge in Insulating Liquids." IEEE Trans. Electr. Insul. 3 (1): 1-17.

[3] Sumoto, I. 1956. "Climbing of Liquid Dielectrics up along Electrode.” Oyo Buturi. 25 (6): 264-5. (in Japanese)

[4] Pickard, W. F. 1961. "Experimental Investigation of the Sumoto Effect.” J. Appl. Phys. 32 (10): 1888-93.

[5] Pohl, H. A. 1978. Dielectrophoresis, the Behavior of 
Neutral Matter in Nonuniform Electric Fields. Cambridge: Cambridge University Press.

[6] Squires, T. M., and Bazant, M. Z. 2004. "Induced-Charge Electro-Osmosis.” J. Fluid Mech. 509: 217-52.

[7] Romos, A., Morgan, H., Green, N. G., Gonzalez, A., and Castellanos, A. 2005. "Pumping of Liquids with Traveling-Wave Electroosmosis." J. of Appl. Phys Trans. 97: 084906-1-8.

[8] Takashima, T., Hanaoka. R., Ishibashi, R., and Ohtsubo, A. 1998. "I-V Characteristics and Liquid Motion in Needle-to-Plane and Razor Blade-to-Plane Configuration in Transformer Oil and Liquid Nitrogen." IEEE Trans. Electr. Insul. 23 (4): 645-58.

[9] Atten, P., and Seyed-Yagoobi, J. 2003. "Electrohydro-Dynamically Induced Dielectric Liquid Flow through Pure Conduction in Point/Plane Geometry." IEEE Trans. Dielectr. Electr. Insul. 10 (1): 27-36.

[10] Hanaoka, R., Nakamichi, H., Takata, S., Fukami, T., and Kanamaru, Y. 2004. "Distinctive Flow Properties of Liquid-Jet Generated by EHD Pump and Conical Nozzle." IEEJ Trans. FM. 124 (5): 399-406. (in Japanese)

[11] Hanaoka, R., Hosodani, N., Takahasi, I., Takata, S., and Fukami, T. 2007. "Practical Electrode System for EHD Liquid Jet Generation and Properties of Liquid Pumping." IEEJ Trans. FM. 127 (12): 769-76. (in Japanese)

[12] Hanaoka, R., Takahashi, I., Takata, S., Fukami, T., and Kanamaru, Y. 2009. "Properties of EHD Pump with Combination of Rod-to-Rod and Meshy Parallel Plates Electrode Assemblies." IEEE Trans. Dielectr. Electr. Insul. 16 (2): 440-7.

[13] Taylor, G. 1964. "Disintegration of Water Drops in an Electric Field." Proc. Roy. Soc. London Ser. A. 280 (1382): 383-97.

[14] Djairam, D. P. H. F., Morshuis, P. H. F., and Smit, J. J. 2006. "Electro-Spraying without Spraying Nozzles Using Self-adjusting Taylor Cones." In Proc. of the 2006 Intern. Symposium on Electrohydrodynamics (ISEHD), 37-40.

[15] Yarin, A. L., Koombhongse, S., and Reneker, D. H. 2001. "Taylor Cone and Jetting from Liquid Droplets in Electro-Spinning of Nanofibers.” J. of Appl. Phys. 90 (9):
4836-46.

[16] Kirby, A. E., Jebrail, M. J., Yang, H., and Wheeler, A. R. 2010. "Folded Emitters for Nanoelectrospray Ionization Mass Spectrometry." Rapid Commun. Mass Spectrom. 24: 3425-31.

[17] Hanaoka, R., Mizuno, T., Takata, S., Fukami, T., and Anzai, H. 2011. "Dynamic Behavior of EHD Extraction Phenomena of Liquids." IEEJ Trans. FM. 131 (10): 824-31. (in Japanese)

[18] Yabe, A., Mori, Y., and Tsuchikata, K. 1980. "Study on EHD Effect Affecting Evaporation." Trans. of the Japan Society of Mechanical Engineers B 46 (406): 1166-71. (in Japanese)

[19] Asakawa, Y. 1976. "Promotion and Reterdation of Heat Transfer by Electric Field." Nature 261: 220-1.

[20] Seyed-Yagoobi, J. 1999. "Augmentation of Two-Phase and Single-Phase Heat Transfer and Mass Transfer with Electrohydrodynamics in Thermal Equipment." In Proc. of the 13th Intern. Conf. of Dielectric Liquids (ICDL), 378-83.

[21] Ma, W., Zhai, S., Zhang, P., Xian, Y., Zhang, L., Shi, R., Sheng, J., Liu, B., and Wu, Z. 2018. "Research Progresses of Flash Evaporation in Aerospace Applications.” Intern. J. of Aerospace Engineering, Article ID 3686802.

[22] Rycroft, M. 2014. "Vegetable Oil as Insulating Fluid for Transformers." Energize (April): 37-40.

[23] Yabe, A., Mori, Y., and Hijikata, K. 1987. "Heat Transfer Enhancement Techniques Utilizing Electric Fields.” In Heat Transfer in High Technology and Power Engineering, edited by Yang, W. J., and Mori, Y. Washington: Hemisphere, 394-405.

[24] Takano, K., Tanasawa, I., and Nishio, S. 1991. "Enhancement of Evaporation of Droplet Using EHD Effect." Trans. of the Japan Society of Mechanical Engineers B 57 (534): 693-8. (in Japanese)

[25] Takano, K., Tanasawa, I., and Nishio, S. 1991. "Enhancement of Evaporation of Droplet Using EHD Effect: 2nd Report, Onset of Instability of Gas-Liquid Interface under Electric Field." Trans. of the Japan Society of Mechanical Engineers B 57 (544): 4216-22. (in Japanese) 